\title{
3D Printed Polymer Photonic Topological Insulators and their Robustness to Structural Disorder
}

Michael S. Mattei ${ }^{1}$, Boyuan Liu ${ }^{2}$, Gerardo A. Mazzei Capote ${ }^{3}$, Zijie Liu ${ }^{3}$, Brandon G. Hacha ${ }^{1}$, Tim A. Osswald ${ }^{3}$, Zongfu $\mathrm{Yu}^{2}$, Randall H. Goldsmith*1

M. S. Mattei, R. H. Goldsmith

Department of Chemistry, University of Wisconsin-Madison

1101 University Avenue, Madison, WI, 53706, USA

E-mail:rhg@chem.wisc.edu

B. Liu, Z. Yu

Department of Electrical and Computer Engineering, University of Wisconsin-Madison

1415 Engineering Drive, Madison, WI, 53703, USA

G. A. Mazzei Capote, Z. Liu, T. A. Osswald

Department of Mechanical Engineering, University of Wisconsin-Madison

1513 University Avenue, Madison, WI, 53703, USA

Keywords: Topological Photonics, Additive Manufacturing, High-Speed FDTD

Abstract: Photonic topological insulators have emerged as an exciting new platform for backscatter-free waveguiding even in the presence of defects, with applications in robust longrange energy and quantum information transfer, spectroscopy and sensing, chiral quantum optics, and optoelectronics. We demonstrate a design for spin-Hall photonic topological insulators with remarkably low refractive index contrast, enabling the synthesis of photonic topological waveguides from polymeric materials for the first time. Our design is compatible with additive manufacturing methods, including fused filament fabrication for microwave frequencies, and constitutes the first demonstration of a 3D printed all-dielectric photonic topological insulator. We combine rapid device fabrication through 3D printing with high-speed FDTD simulation to quantify topological protection of transmission through "omega" shaped bent topological waveguides and find that one corner in the waveguide is 3-5 times more robust to disorder than the other. This dichotomy, a new empirical design rule for $\mathbb{Z}_{2}$ topological insulator devices, is shown to originate in the fundamental system symmetries and is illustrated via the distributions of Poynting vectors that describe energy flow through the waveguide. Taken together, our demonstration of 3D printed polymeric spin-Hall photonic topological insulators paired with quantification of robustness to disorder at bent topological interfaces provides a rapid, flexible scheme for engineering high-performance topological photonic devices across multiple frequency regimes from microwave to $\mathrm{THz}$, to visible.

\section{Introduction}

Photonic topological insulators (TIs), periodic structures with a band gap in the bulk but gapless dispersion at the edges, possess the unique ability to guide light around defects and sharp turns without scattering. ${ }^{[1-4]}$ Such topological protection of light propagation provides an exciting new platform for on-chip low-threshold lasing, ${ }^{[5-9]}$ robust long-range quantum information and energy transfer, ${ }^{[10-12]}$ unidirectional emission from spin-polarized quantum states, ${ }^{[13]}$ low-dimensional 
confinement, ${ }^{[14,15]}$ robust optical resonators and delay lines, ${ }^{[16,17]}$ soliton generation, ${ }^{[18,19]}$ hybrid light-matter states, ${ }^{[20,21]}$ generation of twisted light, and quantum networks. ${ }^{[10,22,23]}$ Photonic TIs were first demonstrated at microwave frequencies by breaking time-reversal symmetry in a lattice of gyromagnetic rods, producing the photonic analogue of the quantum Hall effect ${ }^{[24]}$ which produces a single unidirectional chiral edge state. The absence of a counter-propagating mode ensures that the edge state is robust against backscattering defects. Achieving all-dielectric designs for photonic TIs has required mimicking the spin-Hall, ${ }^{[25-27]}$ and valley-Hall effects. ${ }^{[17]}$ In such systems, two counterpropagating helical edge states exist which are decoupled via their opposite pseudo-spin and valley angular momenta, respectively. Thus, the helical edge states in spin and valley Hall systems are only robust against perturbations which preserve the spin degree of freedom. ${ }^{[16]}$ Indeed, in the only experimental example of quantifying topological protection in dielectric bent topological waveguides, there is still a small amount of measurable loss due to reflection from the corners. ${ }^{[28]}$

Despite their reduced topological protection, all-dielectric photonic TIs show great promise in applications such as low-threshold lasing, ${ }^{[5-8]}$ quantum networks, ${ }^{[9,21,22}$ the generation of twisted light, ${ }^{[29,30]}$ and topological resonator-based sensing, spectroscopy, and strong coupling. ${ }^{[8,21,31]}$ Each of these applications can take advantage of the unique ability of TIs to guide light around sharp turns, for which the influence of fabrication disorder has not yet been quantified. Further, many of these applications also require interfacing quantum emitters with topological photonic systems, which requires good spatial overlap between the photonic mode and the emitter and can be difficult to achieve due to reliance on evanescent tails. Here, we address both of these barriers through a combination of additive manufacturing and rapid simulation techniques. Though we demonstrate our approach and quantify topological protection in bent topological interfaces in the microwave regime, the approach is general across multiple length and energy scales and offers new functionalities for producing photonic TIs and integrating quantum emitters.

We first introduce a design for $\mathbb{Z}_{2}$ pseudo-spin Hall photonic TIs with remarkably low refractive index contrast, thus enabling the many advantages of 3D printing photonic TIs with polymeric materials for the first time. Additive manufacturing (AM) has a highly successful track record in production of photonic materials across multiple length scales from micrometers to centimeters, spanning frequency regimes from optical to microwave. ${ }^{[32-36]}$ Our design for polymeric photonic TIs is readily scalable to any of these length scales through the appropriate choice of AM technique using conventional AM materials. Further, because many materials can easily be doped directly into polymers, our design is ideal for direct integration of quantum emitters into TIs at optical, terahertz, and microwave frequencies. We showcase our design at microwave frequencies using fused filament fabrication (FFF) and demonstrate robust transmission through an "omega" shaped bent topological waveguide.

Using high-speed finite-difference time-domain (FDTD) simulations and supporting experimental data, we also present the first quantitative investigation into the impact of geometric disorder on the transmission through a bent topological waveguide. For all-dielectric topological systems without time reversal symmetry breaking due to magneto-optical effects, it is important to quantify the impact of disorder on the transmission through bent waveguides and resonators in order to optimize device performance. ${ }^{[37,38]}$ We screened 4096 possible corner geometries for such waveguides, and correlated the transmission with the degree of disorder at the corners. Remarkably, we found that the two distinct types of $120^{\circ}$ turn in our bent waveguide structure display a dramatic difference in robustness to disorder, with disorder leading to as much as $\sim 57 \%$ loss at the corner composed mostly of unit cells from the topological lattice (majority-topological), 

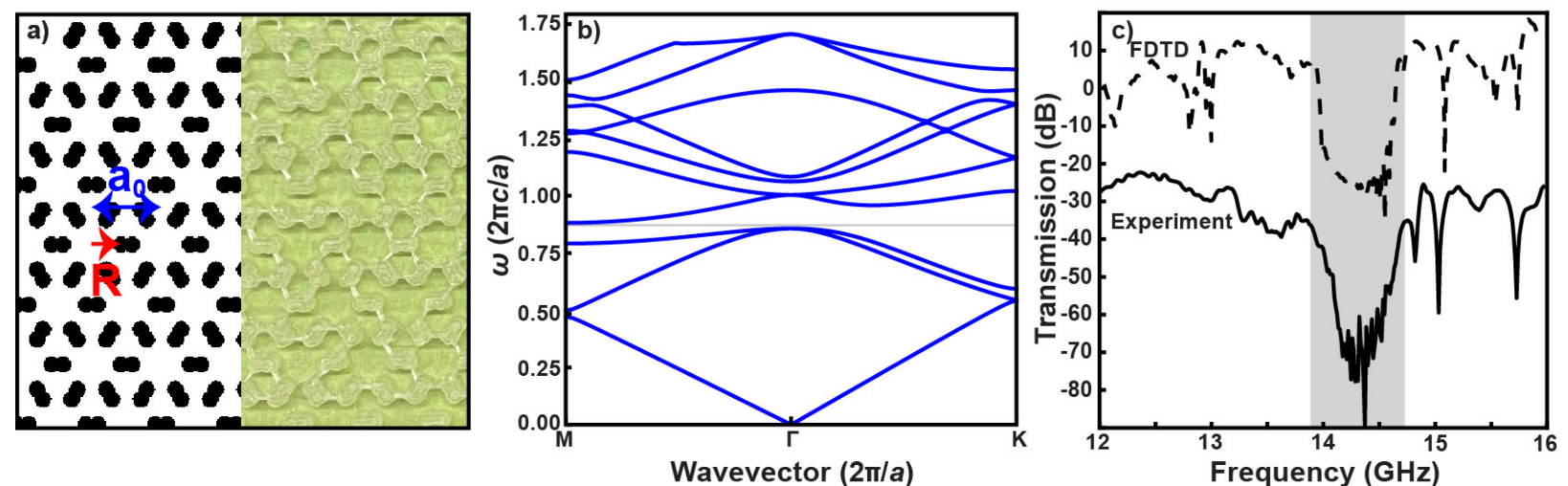

Figure 1. (a) Structure of the expanded honeycomb photonic topological insulator explored in this work, with the 3D printed structure overlaid on the right. (b) Band structure of an infinite honeycomb TI lattice. The complete topological band gap is highlighted in grey. (c) Theoretical and experimental transmission spectra of a finite (24x15 unit cells) expanded honeycomb structure along the $\Gamma$-M direction, with the band gap region highlighted in grey. 
and only $\sim 12 \%$ at the majority-trivial corner. Thus, in devices requiring sharply bent topological interfaces, such as topological resonators, we present a new design rule for choosing corner geometries to optimize robustness to disorder.

\section{Results and Discussion}

We first present a design for two-dimensional (2D) spin-Hall photonic TIs with low refractive index contrast (as low as $n=1.55$ when contrasted with air), greatly expanding the range of available materials for fabricating photonic TIs, including polymers. Polymeric materials provide a number of advantages as media for topological photonics including (often) low absorption at visible wavelengths, straightforward doping of quantum emitters and gain materials into the topological lattice, application in flexible photonic devices, ${ }^{[39]}$ and especially, compatibility with rapid fabrication techniques such as additive manufacturing and soft lithography. Our design (Figure 1a) is an adaptation of the expanded honeycomb lattice of rods proposed by $\mathrm{Wu}$ and $\mathrm{Hu},{ }^{[25]}$ with the rod dimensions and degree of expansion carefully tuned to maintain a complete topological band gap with low index contrast. At a ratio $\mathrm{a}_{0} / \mathrm{R}$ equal to 3 , where $\mathrm{a}_{0}$ is the lattice constant and $\mathrm{R}$ is the distance from the center of the unit cell to the center of a rod, the honeycomb lattice supports a Dirac point between bands 3 and 4 . Decreasing $\mathrm{a}_{0} / \mathrm{R}$ opens the Dirac point into a topological band gap, while increasing the ratio yields a trivial bandgap.

We found complete photonic band gaps for structures with refractive index as low as $\mathrm{n}=1.55$ with $\mathrm{a}_{0} / \mathrm{R}=2.4$ for the topological lattice (Figure 1a,b) and $\mathrm{a}_{0} / \mathrm{R}=4.4$ for its trivial counterpart (Figure S1), when the rod radius is set to $\mathrm{r}=0.12 \mathrm{a}_{0}$. In contrast, previously demonstrated photonic topological insulators have been fabricated from materials with much higher index, such as silicon ${ }^{[27]}(n=3.8)$ and silicon nitride $(n=2.0)^{[40]}$ in the visible and alumina at microwave frequencies $(n=3.1) .{ }^{[41]}$ Our design thus greatly expands the pallet of available materials for producing photonic topological insulators, including polymers at optical, infrared, and microwave frequencies, and oxides at optical frequencies. Further, because this design is compatible with additive manufacturing, it is easily scalable to $\mathrm{THz}^{[36,42]}$ (via stereolithography) and visible ${ }^{[27,43]}$ frequencies (via multiphoton absorption polymerization), for which fabrication is otherwise challenging.

Using FFF, we fabricated the expanded structure $\left(\mathrm{a}_{0}=17.8 \mathrm{~mm}, 7.35 \mathrm{~mm}\right.$ height, Figure 1a overlay) from polylactic acid (PLA) on an aluminum substrate. FFF provides a rapid, automated and highly reproducible means of fabricating complex geometries from thermoplastic polymeric materials, allowing straightforward introduction of arbitrary defects into the structure. FFF also provides a tradeoff between print time and part density (which affects refractive index) by tuning the infill percentage. For PLA (bulk $\mathrm{n}=1.64$ ), maintaining a refractive index above 1.55 for reasonable print times was a key technical challenge in this work. We have selected slicing parameters (Table S1) which result in print times of $\sim 30$ largely unsupervised hours while achieving a refractive index of $\sim 1.61$, as determined from comparison to the simulated transmission spectrum. Specialty filaments with high refractive index ${ }^{[44,45]}$ could be used to produce structures with lower infill percentage and thus lower density, further reducing print times and providing a flexible means for introducing refractive index gradients as a degree of freedom in device design.

We then measured the transmission along $\Gamma-\mathrm{M}$ (propagation along the lattice vector) using a parallel plate waveguide apparatus and observed a $\sim 40 \mathrm{~dB}$ dip in the spectrum at $\sim 14.35 \mathrm{GHz}$ corresponding to the topological band gap, in agreement with high-speed FDTD simulation 

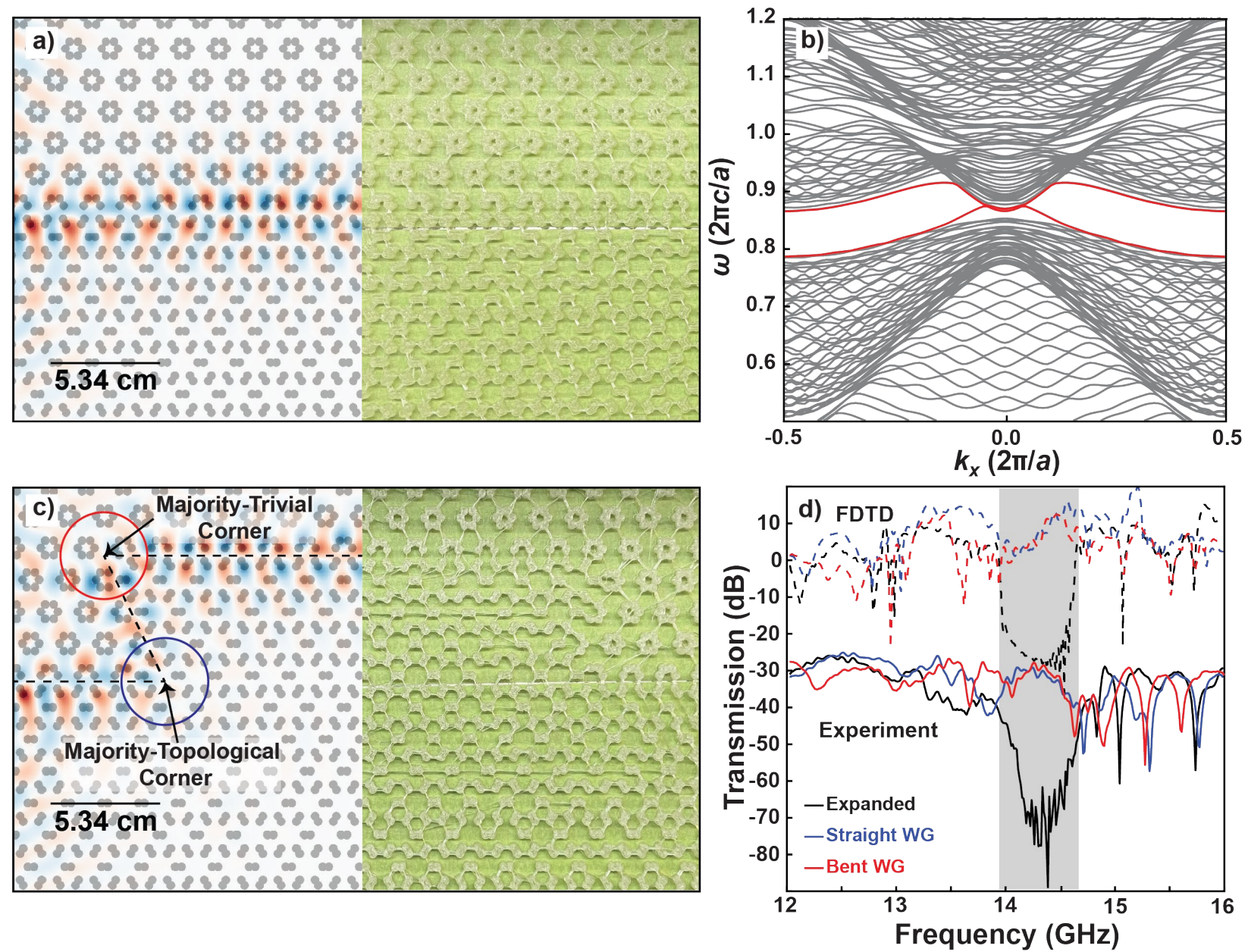

Figure 2. (a) The straight interface between the topological and trivial domains hosts a topological edge state. The $E_{Z}$ component of the edge state shows that the mode is well confined to the straight waveguide. The 3D printed structure is overlaid on the right side. (b) Calculated supercell band structure for the straight waveguide in (a), depicting the gapless topological edge states in red. (c) The topological edge states propagate around sharp turns in a bent "omega" waveguide without backscattering. The black dashed line highlights the boundary between the topological and trivial photonic crystals. (d) Simulated and experimental transmission spectra showing high transmission through both the straight and bent topological waveguides in the band gap region (highlighted in grey). 
(Figure 1c). The interface between the topological and trivial structures supports a topological edge state which bridges the band gap. The interface between our topological and trivial structures is depicted in Figure 2a with the electric field $\left(E_{z}\right)$ distribution of the topological edge state overlaid, and the corresponding band structure is shown in Figure $\mathbf{2 b}$ with the edge states shown in red. These edge states are topologically protected against backscattering from defects which preserve the angular momentum of the wave and should thus navigate sharp turns in the interface. Figure 2c shows a bent "omega" waveguide, with the corresponding topological edge states navigating a Z-shaped turn without loss. Indeed, experimental transmission measurements show high transmission across the entire band gap region, with comparable transmission for the straight and bent waveguides, indicative of topological protection and in excellent agreement with high-speed FDTD simulations (Figure 2d). This constitutes the first experimental example of a fully polymeric photonic topological insulator.

Immunity to defects is a desirable feature of topological systems, and we wanted to quantify this feature of our system. A systematic investigation of immunity to disorder has previously been performed for straight topological waveguides, ${ }^{[38]}$ but the impact of disorder on bent waveguides has not yet been explored, despite use of transmission through bent waveguides as evidence of topological protection. Our bent waveguide has two unique pairs of corners, each of which is a combination of the topological and trivial unit cell. To investigate the impact of disorder at the corners, we investigated all possible geometries in which the 6 rods at each corner can occupy either their position in the trivial lattice or their position in the topological lattice. Thus, there are $2^{12}=4096$ possible corner configurations in this large but discrete parameter sweep. We simulated the transmission through all 4096 possible structures using a commercial high-speed FDTD solver ${ }^{[46]}$ (Tidy3D) with a total simulation time of 14.8 hours (13 seconds per simulation). We timed a 2D simulation using conventional FDTD software with the parameters set as closely as possible to those used in our Tidy3D simulations and measured a simulation time of 5.81 minutes. Running 4096 such simulations would thus require 2.4 weeks of simulation time (a $24 \mathrm{x}$ increase).

A summary of the transmission simulations through all sampled configurations is shown in Figure 3. The bent waveguide design in Figure 2 has corner configurations similar to those used in previous works, and constitutes a small enough perturbation of the $\mathrm{C}_{6}$ symmetry of the bulk lattice to maintain topologically protected propagation. ${ }^{[47,48]}$ This structure is thus an intuitive benchmark to which we can compare the disordered structures, since it contains only complete unit cells from the topological and trivial lattice. To compare the transmission of all simulated structures, we calculated the percent decrease in transmission relative to the structure with the highest transmission, integrating across the entire bandgap region. Figure 3a shows a histogram of the percent decrease in transmission through all 4096 simulated topological waveguides. The distribution is continuous and appears to be multimodal. The multimodal nature of the distribution likely arises from the fact that we have only considered two discrete possible positions for each rod, and thus a discrete number of possible transmission values for each corner. More striking is the large spread in transmission values (a range of 73\%), which suggests that a majority of the simulated geometries induce significant backscattering, and thus do not preserve the spin angular momentum of the wave. This behavior is consistent with the "weak" topological protection predicted for pseudo-spin Hall TIs. ${ }^{[38,49,50]}$ Additionally, there are three simulated structures with slightly $(<1 \%)$ higher total trans mission compared to the design in Figure 2. However, the increase in transmission is well within the uncertainty of our experimental measurements and will thus not be explored further in this work. 

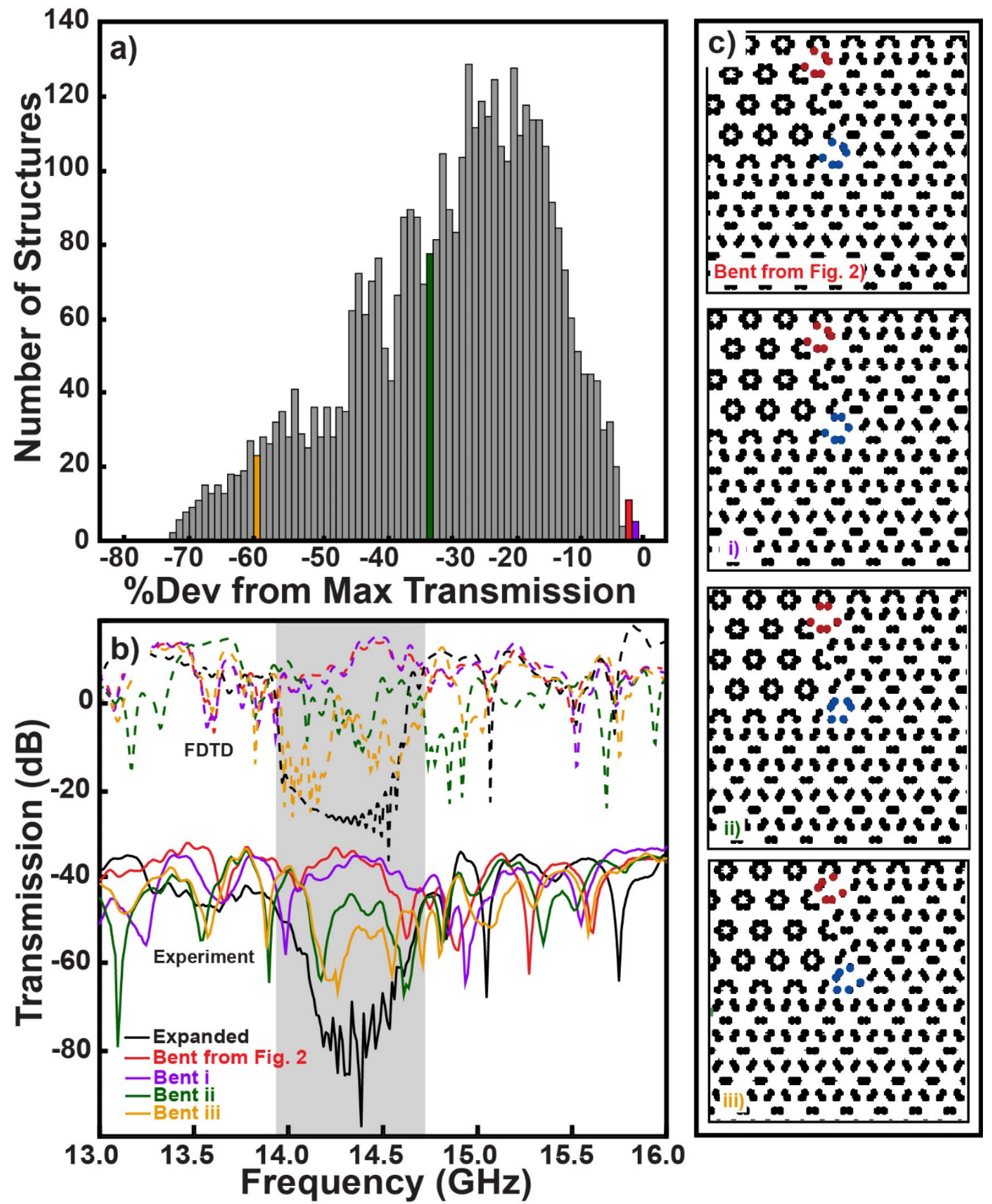

Figure 3. (a) Histogram of the percent deviation (linear scale, from simulation) for the total transmission through all 4096 simulated bent waveguide structures relative to the structure with the highest total transmission. The position of each structure is color coded in the histogram. The red bar indicates the transmission of the original bent waveguide from Figure 2. The other colored bars correspond to the structures in part b) (b) Simulated and experimental transmission spectra for the 4 example structures in (c). The experimental spectra of show Structures (i-iii) in order of decreasing transmission, in agreement with the theoretically predicted trend. 
In Figures 3b and 3c, we select three examples of disordered structures from throughout the distribution. Experimentally measured spectra for these three structures (Figure 3b) show excellent agreement with FDTD and follow the expected trend predicted by the theory, with Structure (i) showing the highest transmission and Structure (iii) the lowest. This rapid production and experimental verificatio $\mathrm{n}$ of topological photonic materials via additive manufacturing thus represents a powerful new tool for exploring topological protection in pseudo-spin Hall systems. Further examples of disordered structures with various transmission are shown in Figure S2. A more quantitative treatment of transmission as a function of disorder is necessary to draw practical conclusions from the histogram in Figure 3a and is presented below.

Methods for quantifying disorder in one dimension have previously been explored for quantifying topological protection for different degrees of 1D Anderson localization in straight TI waveguides. ${ }^{[38]}$ However, because our disordered corners are 2D distortions localized on single unit cells, we cannot follow an analogous strategy to that outlined in reference 38 . To quantify the disorder in each simulated waveguide, we treated the two corners individually and adopted a simple method based on the percent deviation of each corner geometry from the original structure in Figure 2. For each disordered corner configuration, we defined a square enclosing only the corner unit cell and simply subtracted the resulting image from the corresponding image for the original bent waveguide structure. By integrating the absolute value of the subtracted image and normalizing to the number of pixels in the image, we obtained a measure of the percent deviation relative to the ordered structure. A schematic of this process is shown in Figure S3.

When analyzing a particular corner configuration, that individual corner geometry is represented in $2^{6}=64$ different waveguide structures corresponding to all possible configurations of the other corner. Thus, for each individual corner configuration, we calculated an average and standard deviation of the percent transmission through all 64 structures in which that corner configuration is present. Figures $\mathbf{4 a}$ and $\mathbf{4 b}$ show the averaged percent decrease in transmission as a function of disorder for the top and bottom corners in the bent waveguide, respectively. We note that lattice around the top corner is composed mostly of unit cells from the trivial lattice, while the lattice surrounding the bottom corner is mostly topological. We will thus refer to these two corners as "majority-trivial" (top) and "majority-topological" (bottom), respectively, Figure 2c. The average transmission decrease due to disorder in the majority-trivial corner shows a very slight downward trend with increasing disorder, with a $12 \%$ difference between the maximum and minimum transmission values, a pronounced robustness to disorder. In contrast, the corresponding plot for the majority-topological corner reveals a much stronger downward trend, with a range in average transmission values of 58\%. The configuration used for the majority-trivial corner in our bent waveguide is thus substantially more robust to disorder compared to the majority-topological (by a factor of 4.8). This dichotomy is further evidenced by the fact that the standard deviations in Figure 4a are larger than those in Figure $4 \mathbf{b}$ by a factor of 3 . In Figure 4a, the standard deviation for each configuration of the majority-trivial corner reflects the influence of disorder in the majority-topological corner, and vice versa for Figure $\mathbf{4 b}$. Therefore, the large (up to 25\%) standard deviations observed in Figure 4a also demonstrate that transmission through the bent waveguide is less robust to disorder in the majority-topological corner than in the majority-trivial corner. To verify that this behavior is not an artifact of the relatively short diagonal length, we repeated this robustness analysis for an omega waveguide with more unit cells along the diagonal (Figure S4) and observed the same relative robustness for the two corners. We also confirmed that shifting the input direction of the wave does not flip the behavior of the corners: in both cases, the 
majority-topological corner is less robust, consistent with the expectation that the structure obeys optical reciprocity (Figure S5).

The asymmetry between the corners seems strange when compared to conventional waveguides or even Chern TIs, but is readily explained by considering the nature of topological protection in these $\mathbb{Z}_{2}$ pseudo-spin Hall TIs. Here, the bulk topology arises from the $C_{6}$ symmetry of the lattice, which is broken at the interface. ${ }^{[25]}$ Thus, there is thus no true bulk-edge correspondence in these crystals, ${ }^{[49]}$ and the broken $C_{6}$ symmetry at the interface leads to a small band gap between the edge states. ${ }^{[25]}$ This detail is the origin of the "weak" topological protection in $\mathbb{Z}_{2}$ pseudo-spin Hall TIs. Asymmetry is easily seen in Figure 4c, where we have overlaid the mode profile with a hexagonal grid depicting the local point group symmetry of each unit cell. Each unit cell along the interface is outlined in yellow and belongs to the $C_{2 v}$ point group, which does not have $C_{6}$ symmetry. All other unit cells are outlined in black and belong to the $D_{6 h}$ point group, which does possess $\mathrm{C}_{6}$ symmetry. Though the mode largely resides in the interfacial $C_{2 v}$ cells as expected, when evanescently leaking into $D_{6 h}$ cells it clearly favors the topological lattice over the trivial lattice. This behavior is consistent with the observations of $\mathrm{Wu}$ and $\mathrm{Hu},{ }^{[25]}$ as in pseudo spin-Hall TIs, energy flow at the ideal interface largely resembles the topological bulk lattice rather than the trivial phase. Thus, the mode is more accurately thought of as spanning the interfacial and topological lattice, and therefore the corners should no longer be expected to be equivalent. The $D_{6 h}$ cells in the trivial lattice do not offer protection from scattering at defects because the circulating Poynting vector in each trivial unit cell does not lead to net propagation at the boundaries. In contrast, the $D_{6 h}$ cells in the topological lattice offer protection because the circulation of the Poynting (pseudo-spin) is not confined to individual unit cells, leading to edge propagation. ${ }^{[25]}$ At the more robust majority-trivial corner, the mode is clearly seen partially occupying a topological $D_{6 h}$ cell, thus still benefiting from this topological protection, Figure 4c. In contrast at the more sensitive majority-topological corner, the mode is contained almost entirely in the interfacial $C_{2 v}$ cells that lack $\mathrm{C}_{6}$ symmetry, where protection from disorder is minimal, Figure 4c.

This prediction of a fundamental asymmetry between the corners that underlies the behavior in Figure 4a,b is dramatically visualized in Figure 5, which depicts a stream plot of the Poynting vectors overlaid on the bent topological waveguide. Near the more robust majority-trivial corner, the bulk of the Poynting vectors (densely spaced blue lines) from the lower part of the waveguide are clearly seen to essentially avoid the $C_{2 v}$ unit cell (orange circle) and flow around the bend through the adjacent topological unit cell (black circle) facilitating robust propagation resistant to interfacial defects at the corner (red highlighted sites). In contrast, the Poynting vectors near the more sensitive majority-topological corner (highlighted by the blue arrow) show that the mode largely propagates thro ugh the $C_{2 v}$ unit cell (green circle) reducing the topological protection due to the lack of local $\mathrm{C}_{6}$ symmetry. Said another way, transmission through the topological waveguide can be thought to be insensitive to defects in the majority-trivial corner (red highlighted sites) because the energy flow essentially avoids them, whereas defects in the majority-topological corner (blue highlighted sites) are damaging to transmission (Figure 4a) because energy flows directly through these sites. Based on our analysis, we estimate that the majority-trivial corner of our bent waveguide is 3 to 5 times more robust to geometric disorder than that used in the majoritytopological corner and thus offer a new empirical design rule for pseudo-spin Hall TI-based bent waveguides, and a more practical geometry for use in photonic devices such as resonators.

We end by comparing the behavior of our pseudo-spin Hall TI waveguide to other waveguides. In conventional trivial waveguides, transmission is typically maximized by 

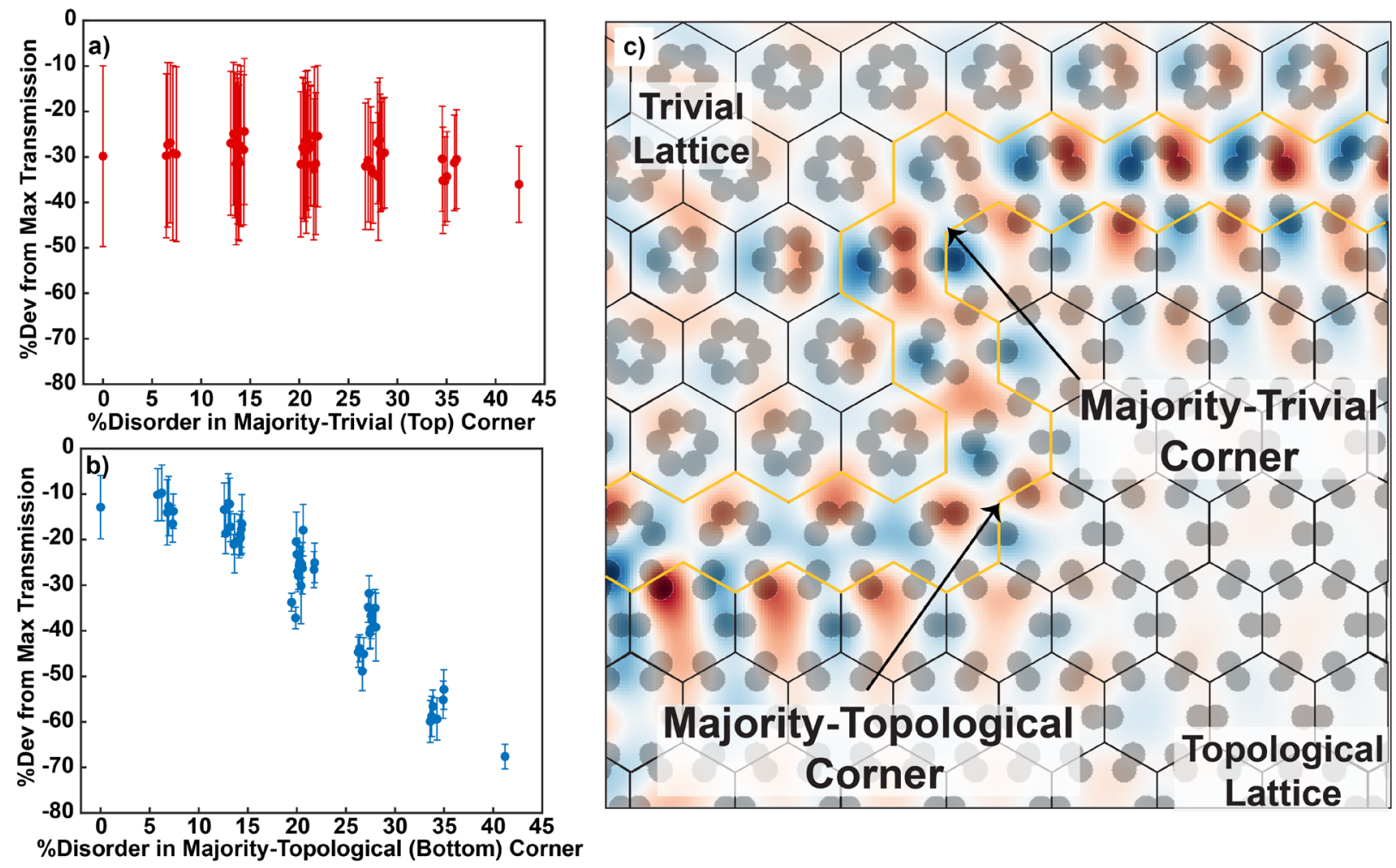

Figure 4. Decrease in transmission through the bent topological waveguides simulated in Figure 3 as a function of disorder at the majority-trivial (a) and majority-topological (b) corner. Each corner configuration is present in 64 different structures (paired with each of the 64 configurations of the other corner). Each point in the plot thus shows the average and standard deviation of the transmission through 64 structures. (c) Hexagonal lattice indicating local symmetry overlayed on the bent topological waveguide. Unit cells outlined in yellow belong to the $C_{2 v}$ point group which lacks $C_{6}$ symmetry, while those outlined in black belong to the $D_{6 h}$ point group which possesses $C_{6}$ symmetry. 


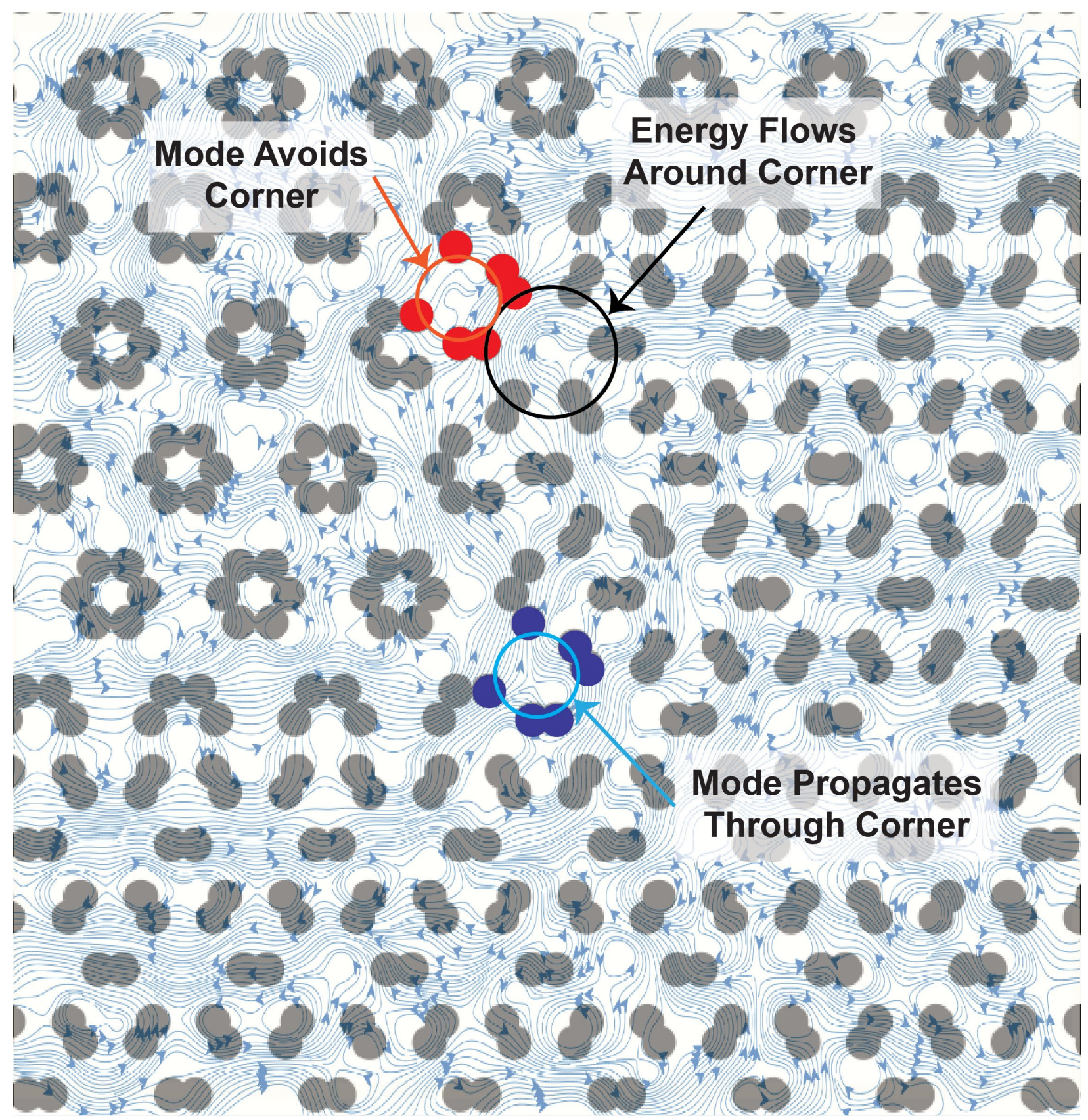

Figure 5. Poynting vector stream plot showing the flow of energy through our bent topological waveguide. At the majority-trivial corner (red dots), the mode largely avoids the $C_{2 v}$ interfacial unit cell (orange circle) and instead circulates around the adjacent $D_{6 h}$ topological unit cell (black circle). In contrast, at the majority-topological corner (blue dots), the mode propagates directly through the $C_{2 v}$ interfacial unit cell (blue circle and dots) and is therefore less robust to disorder at these positions. 
minimizing leakage of the optical mode out of the core and into the cladding, while here in the pseudo-spin Hall TI, the protection is enhanced by leakage into the topological "cladding" and reduction of density in the symmetry-lacking interfacial "core." In a true Chern TI, the bulk-edge correspondence principle would likely offer protection equally at both corners, since backscatterimmune edge states are guaranteed by the bulk topology regardless of local lattice symmetry at the interface. ${ }^{[1,2,49]}$

\section{Conclusions}

We have developed a design for polymeric $\mathbb{Z}_{2}$ pseudo-spin Hall photonic topological insulators with remarkably low refractive index contrast (as low as 1.55). Our design is compatible with and realized by additive manufacturing techniques, greatly expanding the materials pallet for photonic TIs and allowing rapid prototyping of photonic devices with flexible design and the possibility of easily intercalating emitters. Experimental and simulated transmission spectra of Tis and interfacial waveguides offer agreement. We combined our rapid fabrication capability with highspeed FDTD simulation to screen 4096 possible corner configurations for a bent topological waveguide. By quantifying loss in transmission as a function of disorder at two distinct corners in a bent topological waveguide, we observed an asymmetry where one corner is 3-5 times more robust to disorder than the other. This dichotomy originates in the fundamental symmetries of the lattice and constitutes a new empirical design rule for photonic TI-based light-guiding devices such as resonators and delay lines. We also note that high-speed simulation, when combined with additive manufacturing can provide a powerful platform for exploring and optimizing novel designs for topological photonics by generating training sets of experimental data for machine learning. Combined, the realization of polymeric photonic TIs and quantification of the topological protection at sharp turns are additional steps forward for implementation of photonic TIs in quantum information science, low-threshold lasing, sensing and spectroscopy, and strong lightmatter interactions across energy scales from visible to microwave.

\section{Experimental Section}

\subsection{Band Structure and Transmission Calculations}

Band structures of the 2D topological and trivial band gap structures were performed using the MIT Photonic Bandgap (MPB) Python package, treating both structures as an infinite lattice. ${ }^{[51]}$ The band structure for the straight waveguide was also calculated using MPB, treating the structure as an infinite lattice along the waveguide (X) axis and a finite supercell (10 topological unit cells and 10 trivial unit cells) along the $\mathrm{Y}$ axis.

In-plane transmission simulations were performed using Tidy3D (Flexcompute, Inc.) with a point source polarized along $\mathrm{Z}$ (perpendicular to the plane of the crystal). The $\mathrm{X}$ and $\mathrm{Y}$ boundary conditions were set to the built-in 'absorber' option, and the $\mathrm{Z}$ boundary condition was periodic. A perfect electrical conductor (PEC) layer was placed above and below the crystal to approximate the parallel plate waveguide. The high-accuracy simulations compared with our experimental measurements were performed using 128 mesh cells per unit cell along X and Y, and 3 mesh cells along $\mathrm{Z}$ (one mesh cell for each PEC layer and one for the crystal, effectively a 2D simulation). The 4096 high-speed simulations for optimizing corner geometry were performed using 32 mesh cells per unit cell along $\mathrm{X}$ and $\mathrm{Y}$, and one mesh cell each for the crystal and PEC layers along Z. The bent waveguide structure possesses mirror symmetry across the plane perpendicular to the propagation axis. For the corner optimization process, we therefore simulated only the first half of 
the bent waveguide to decrease simulation time. All transmission spectra were acquired by measuring the electric field amplitude at a time-domain monitor and taking the Fourier transform and normalizing by subtracting the simulated spectrum with no crystal present.

\subsection{Additive Manufacturing of Microwave Topological Insulators}

An FFF system (FusedForm FF600) was used to 3D print our topological insulator samples from polylactic acid (Matterhackers "Natural PLA PRO", $1.75 \mathrm{~mm}$ diameter) using a $0.8 \mathrm{~mm}$ nozzle and a layer height of $0.2 \mathrm{~mm}$. Care was taken to ensure that all the acquired spools of filament came from the same lot to avoid variations in material properties from sample to sample. All samples were printed on 1/16-inch-thick aluminum substrates, which do not influence the transmission through the sample. Green painter's tape (3M Scotch 401+ Masking Tape) was applied to the aluminum sheet prior to printing to improve adhesion of the PLA to the substrate. Toolpath generation was performed using the Simplify3D slicing engine (v4.1.2). The slicing parameters (Table S1) were selected iteratively to maximize the refractive index of the printed part while maintaining fidelity of the geometric features and constraining print times to 33 hours. Shorter print times can in principle be achieved by using custom high-index thermoplastic materials and decreasing the infill percentage. ${ }^{[44,52]}$ Common defects that arise due to the printing process included polymer drool between adjacent structures, unwanted z-height deposition that resulted in inconsistent height of the structure, voids in the areas where contiguous circular beads meet, as well as warpage. These issues were mitigated through manipulation of the slicing engine parameters, resulting in the structures used in this work.

\subsection{Transmission Measurements of Microwave Topological Insulators}

In-plane transmission measurements of microwave TIs were performed in a parallel plate waveguide using a Keysight E5071C network analyzer with antennas from Fairview Microwave (SKU: SC3792). The sample is surrounded by radiofrequency absorbing foam and placed between two aluminum plates. The antennas are fixed to the top aluminum plate and spaced $35.8 \mathrm{~cm}$ apart along the long axis of the waveguide so that both antennas reside inside the photonic crystal (Figure S6).

\section{Supporting Information}

(Figure S1) Geometry, band structure, and simulated and experimental transmission plots for the trivial (compressed) honeycomb lattice. (Figure S2) Additional examples of simulated corner geometries for the bent waveguide. (Table S1) List of slicing parameters used in FFF. (Figure S3) Illustration of the procedure used to determine the degree of deviation of each corner geometries from the original bent structure. (Figure S4) Corner robustness analysis for a taller omega waveguide. (Figure S5) Simulation of forward and backward propagation through a Z-shaped topological waveguide to verify reciprocity. (Figure S6) Photographs of the parallel plate waveguide apparatus.

\section{Acknowledgments}

This research was supported by the National Science Foundation through the University of Wisconsin Materials Research Science and Engineering Center (DMR-1720415). The authors thank Professor Nader Behdad, Professor Yin Poo, Sachin Vaidya, and Steve Myers for helpful discussions of the parallel plate waveguide transmission measurements. 


\section{Conflict of Interest}

The authors declare no conflict of interest.

\section{References}

[1] L. Lu, J. D. Joannopoulos, M. Soljačić, Nat. Photonics 2014, 8, 821.

[2] A. B. Khanikaev, G. Shvets, Nat. Photonics 2017, 11, 763.

[3] T. Ozawa, H. M. Price, A. Amo, N. Goldman, M. Hafezi, L. Lu, M. C. Rechtsman, D. Schuster, J. Simon, O. Zilberberg, I. Carusotto, Rev. Mod. Phys. 2019, 91, 015006.

[4] Y. Wu, C. Li, X. Hu, Y. Ao, Y. Zhao, Q. Gong, Adv. Opt. Mater. 2017, 5, 1700357.

[5] M. A. Bandres, S. Wittek, G. Harari, M. Parto, J. Ren, M. Segev, D. N. Christodoulides, M. Khajavikhan, Science. 2018, 359, 1231.

[6] L. Pilozzi, C. Conti, Phys. Rev. B 2016, 93, 195317.

[7] Y. Gong, S. Wong, A. J. Bennett, D. L. Huffaker, S. S. Oh, ACS Photonics 2020, 7, 2089.

[8] A. Dikopoltsev, T. H. Harder, E. Lustig, O. A. Egorov, J. Beierlein, A. Wolf, Y. Lumer, M. Emmerling, C. Schneider, S. Höfling, M. Segev, S. Klembt, Science. 2021, 373, 1514.

[9] Q. Chen, L. Zhang, M. He, Z. Wang, X. Lin, F. Gao, Y. Yang, B. Zhang, H. Chen, Adv. Opt. Mater. 2019, 7, 1900036.

[10] S. A. H. Gangaraj, G. W. Hanson, M. Antezza, Phys. Rev. A 2017, 95, 1.

[11] P. Doyeux, S. A. H. Gangaraj, G. W. Hanson, M. Antezza, Phys. Rev. Lett. 2017, 119, 2.

[12] Y. Wang, J. Ren, W. Zhang, L. He, X. Zhang, Phys. Rev. Appl. 2020, 14, 054007.

[13] J.-W. Dong, X.-D. Chen, H. Zhu, Y. Wang, X. Zhang, Nat. Mater. 2017163 2016, 16, 298.

[14] A. Vakulenko, S. Kiriushechkina, M. Wang, M. Li, D. Zhirihin, X. Ni, S. Guddala, D. Korobkin, A. Alù, A. B. Khanikaev, Adv. Mater. 2021, 33, 2004376.

[15] K.-H. Kim, K.-K. Om, Adv. Opt. Mater. 2021, 9, 2001865.

[16] K. Lai, T. Ma, X. Bo, S. Anlage, G. Shvets, Sci. Rep. 2016, 6, 1.

[17] T. Ma, G. Shvets, New J. Phys. 2016, 18, 025012.

[18] D. Leykam, Y. D. Chong, Phys. Rev. Lett. 2016, 117, 1.

[19] S. Mukherjee, M. C. Rechtsman, Science. 2020, 368, 856.

[20] S. Guddala, F. Komissarenko, S. Kiriushechkina, A. Vakulenko, M. Li, V. M. Menon, A. Alù, A. B. Khanikaev, Science. 2021, 374, 225.

[21] W. Liu, Z. Ji, Y. Wang, G. Modi, M. Hwang, B. Zheng, V. J. Sorger, A. Pan, R. Agarwal, Science. 2020, 370, 600 .

[22] M. C. Rechtsman, Y. Lumer, Y. Plotnik, A. Perez-Leija, A. Szameit, M. Segev, Optica 2016, 3, 925.

[23] P. Lodahl, S. Mahmoodian, S. Stobbe, A. Rauschenbeutel, P. Schneeweiss, J. Volz, H. Pichler, P. Zoller, Nature 2017, 541, 473.

[24] Z. Wang, Y. D. Chong, J. D. Joannopoulos, M. Soljačić, Phys. Rev. Lett. 2008, 100, 013905.

[25] L.-H. Wu, X. Hu, Phys. Rev. Lett. 2015, 114, 223901.

[26] N. Parappurath, F. Alpeggiani, L. Kuipers, E. Verhagen, Sci. Adv. 2020, 6.

[27] S. Peng, N. J. Schilder, X. Ni, J. Van De Groep, M. L. Brongersma, A. Alù, A. B. Khanikaev, H. A. Atwater, A. Polman, Phys. Rev. Lett. 2019, 122, 117401.

[28] S. Arora, T. Bauer, R. Barczyk, E. Verhagen, L. Kuipers, Light Sci. Appl. 2021, 10, 1.

[29] Z. Zhang, X. Qiao, B. Midya, K. Liu, J. Sun, T. Wu, W. Liu, R. Agarwal, J. M. Jornet, S. Longhi, N. M. Litchinitser, L. Feng, Science. 2020, 368, 760.

[30] Z. Q. Yang, Z. K. Shao, H. Z. Chen, X. R. Mao, R. M. Ma, Phys. Rev. Lett. 2020, 125, 013903. 
[31] A. M. Fox, A. P. Foster, E. Clarke, L. R. Wilson, M. J. Mehrabad, M. S. Skolnick, P. K. Patil, R. Dost, Opt.2020, 7, 1690.

[32] A. Camposeo, L. Persano, M. Farsari, D. Pisignano, Adv. Opt. Mater. 2019, 7, 1800419.

[33] F. Kotz, A. S. Quick, P. Risch, T. Martin, T. Hoose, M. Thiel, D. Helmer, B. E. Rapp, Adv. Mater. 2021, 33, 2006341.

[34] J. Köpfler, T. Frenzel, J. Schmalian, M. Wegener, Adv. Mater. 2021, 33, 2103205.

[35] S. A. Rinne, F. García-Santamaría, P. V. Braun, Nat. Photonics 2007, 2, 52.

[36] M. T. A. Khan, H. Li, N. N. M. Duong, A. Blanco-Redondo, S. Atakaramians, Adv. Mater. Technol. 2021, 6, 2100252.

[37] R. E. Christiansen, F. Wang, O. Sigmund, S. Stobbe, Nanophotonics 2019, 8, 1363.

[38] B. Orazbayev, R. Fleury, Nanophotonics 2019, 8, 1433.

[39] Z. Gao, F. Gao, Y. Zhang, Y. Luo, B. Zhang, Adv. Opt. Mater. 2018, 6, 1800532.

[40] W. Liu, M. Hwang, Z. Ji, Y. Wang, G. Modi, R. Agarwal, Nano Lett. 2020, 20, 1329.

[41] X. D. Chen, W. M. Deng, F. L. Shi, F. L. Zhao, M. Chen, J. W. Dong, Phys. Rev. Lett. 2019, 122, 233902.

[42] H. Xiong, Q. Wu, Y. Lu, R. Wang, Q. Zhang, J. Qi, J. Yao, J. Xu, Opt. Express 2019, 27, 22819.

[43] W. Liu, M. Hwang, Z. Ji, Y. Wang, G. Modi, R. Agarwal, Nano Lett. 2020, 20, 1329.

[44] Y. Wu, D. Isakov, P. S. Grant, Materials. 2017, 10.

[45] F. Castles, D. Isakov, A. Lui, Q. Lei, C. E. J. Dancer, Y. Wang, J. M. Janurudin, S. C. Speller, C. R. M. Grovenor, P. S. Grant, Sci. Rep. 2016, 6, 1.

[46] T. W. Hughes, M. Minkov, V. Liu, Z. Yu, S. Fan, Appl. Phys. Lett. 2021, 119, 150502.

[47] B.-Y. Xie, G.-X. Su, H.-F. Wang, H. Su, X.-P. Shen, P. Zhan, M.-H. Lu, Z.-L. Wang, Y.F. Chen, Phys. Rev. Lett. 2019, 122, 233903.

[48] Y. Peng, B. Yan, J. Xie, E. Liu, H. Li, R. Ge, F. Gao, J. Liu, Phys. Status Solidi-Rapid Res. Lett. 2020, 14, 2000202.

[49] M. Saba, S. Wong, M. Elman, S. S. Oh, O. Hess, Phys. Rev. B 2020, 101, 054307.

[50] S. J. Palmer, V. Giannini, Phys. Rev. Res. 2021, 3, L022013.

[51] J. D. Joannopoulos, S. G. Johnson, Opt. Express 2001, 8, 173.

[52] G. A. Mazzei Capote, M. C. Montoya-Ospina, Z. Liu, M. S. Mattei, B. Liu, A. P. Delgado, Z. Yu, R. H. Goldsmith, T. A. Osswald, ChemRxiv 2021, DOI 10.26434/chemrxiv-2021gmm4l. 
For Table of Contents Only

3D Printed Photonic TI

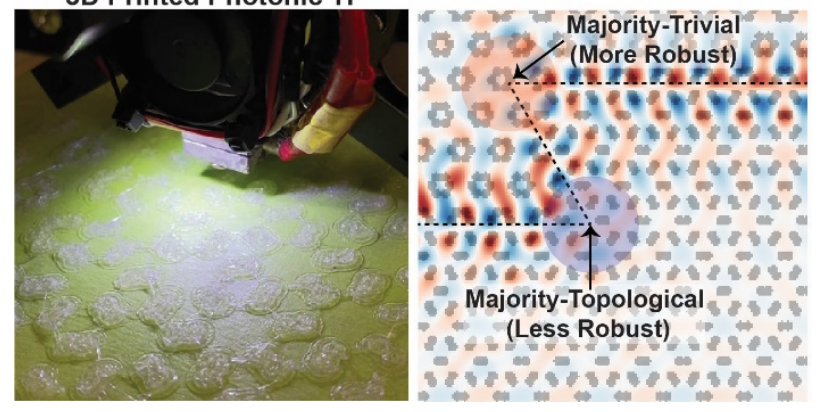

